\title{
Charge Carriers in Commercial Photocatalysts: Fractal Kinetics and Effect of "Inert" Additives
}

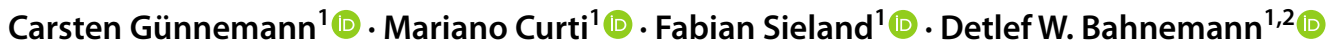

Published online: 12 June 2020

(c) The Author(s) 2020

\begin{abstract}
High photon conversion efficiencies and low-cost materials are crucial for implementing photocatalytic solutions in large scale applications. To improve the conversion efficiencies, knowledge on the lifetime and dynamics of photogenerated charge carriers is fundamental, for which transient absorption spectroscopy has provided important insights. Regarding the cost of the materials, one approach to decrease it is mixing the photocatalyst with cheaper additives while avoiding a significant loss in its activity. In this short review we summarize the findings of our group dealing with both aspects. A new model to fit transient absorption decays, based on fractal kinetics, is discussed. The model has been applied to the transient decays of $\mathrm{TiO}_{2}$ mixtures with binary particle size distributions and of $\mathrm{TiO}_{2}$ mixtures with a priori inert additives. In both cases, the insights obtained from the model were crucial to explain the photocatalytic activities of the mixtures.
\end{abstract}

Keywords Fractal kinetics $\cdot$ Titanium dioxide $\cdot$ Photocatalysis $\cdot$ Transient absorption spectroscopy

\section{Introduction}

Despite extensive research efforts, heterogeneous photocatalysis has yet to achieve a significant deployment in technological applications [1,2]. At the core of this lack of success are the many mechanistic facets still to be unraveled and understood in photocatalytic processes [3]. This has led, expectedly, to a bottleneck in improving the photon conversion efficiencies up to the necessary ranges for the economic feasibility of these processes. Ultimately, the technological success of photocatalytic approaches will be delimited by their benefit-cost ratios [4], from which two obvious optimization avenues emerge: on the one hand, to increase the photon conversion efficiency (i.e. increase benefit); on the

Electronic supplementary material The online version of this article (https://doi.org/10.1007/s11244-020-01282-3) contains supplementary material, which is available to authorized users.

Detlef W. Bahnemann

bahnemann@iftc.uni-hannover.de

1 Institut für Technische Chemie, Gottfried Wilhelm Leibniz Universität Hannover, Callinstrasse 3, 30167 Hannover, Germany

2 Laboratory "Photoactive Nanocomposite Materials", Saint-Petersburg State University, Ulyanovskaya Str. 1, Peterhof, Saint Petersburg, Russia 198504 other, to decrease the cost of preparing and implementing photocatalysts.

To improve the photon conversion efficiencies, as already noted, there is a strong need to deepen our mechanistic knowledge. To this end, numerous techniques can be successfully and complementarily applied to photocatalysts systems. Particularly, our group has focused on the use of transient absorption spectroscopy (TAS) in reflectance mode to study primary processes in photocatalysis [5], i.e. those occurring right after the excitation of the material. Perhaps the most salient feature of this technique is the fact that photocatalyst powders or films can be readily analyzed under realistic conditions, be it in suspension, stand-alone, or under controlled atmospheres. This means that, unlike other analyses that require vacuum or other specific conditions, the conclusions drawn from TAS can be directly transferred to actual photocatalytic reactions. On the downside, the kinetic analysis of transient spectra decays is usually not straightforward. The simplest approach, namely a second-order scheme, presents several limitations, and thus more complex ones must be considered. In particular, our group has developed a modified second-order model that considers the fractal nature of the material, greatly improving the description of the decays. Chemical reactions where the rate constant shows a time dependence are said to exhibit fractal kinetics [6]. From the mathematical point of view, a 
fractal is an object with a fractal dimension that is usually unequal to the classical dimensions of the Euclidean space (1D, 2D, and 3D) [7]. However, fractal kinetics are not limited to reaction media with fractal geometries, but instead commonly occur for diffusion-controlled reactions with geometrical constraints [6], such as electron transfer reactions on rigid glassy matrices [8] or charge carrier recombination on surfaces [9].

Regarding the second optimization avenue, i.e. decrease the cost of preparing and implementing photocatalysts, our group has focused on how different additives affect the activity of commercial photocatalyst powders based on anatase $\mathrm{TiO}_{2}$. There are two aspects to this topic. First, we analyzed the feasibility of mixing these materials with other powders, equally based on anatase, but sold commercially (at a much lower price) for their application as white pigments. Although we expected a sharp decrease in the photocatalytic activity upon adding the pigment, surprisingly it did not display such an effect. Second, we considered how other solids that may be present in real formulations (e.g. $\mathrm{CaCO}_{3}$ or $\mathrm{NaCO}_{3}$ ) could affect the photocatalyst. In this case, we observed a strong effect of the nature of the cation, highlighting possible challenges in the implementation of the photocatalysts.

This short review summarizes our findings on these topics, contextualizing them with a short description of primary processes in photocatalysis and a brief note on the analysis of TAS data. We comment on the benefits of using our fractal-based model to analyze the decays and extend its application to mesoporous $\mathrm{TiO}_{2}$ films of different roughnesses. Finally, we show our work on binary mixtures based on $\mathrm{TiO}_{2}$, both for photocatalyst-pigment and photocatalystadditive compositions, employing once more TAS as a key technique to explain the differing behaviors.

\section{Primary Processes in Photocatalysis}

The excitation of a semiconductor particle leads to the promotion of electrons from the valence band to the conduction band, while holes remain in the valence band (Fig. 1, 1). The photogenerated charge carriers can directly recombine with each other (Fig. 1, 2) or they can be trapped (Fig. 1, 3). The trapping of holes occurs within femtoseconds, which is also the case for the shallow trapping of electrons, while the deep trapping of the latter takes picoseconds [3]. Trapped electrons and holes can take part in chemical reactions with acceptor molecules (Fig. 1, 5) or donor molecules (Fig. 1, 6), respectively. While the most likely fate for shallow trapped charge carriers is recombination (Fig. 1, 4; 1-10 ps), deep trapped charge carriers survive a longer time $(>20 \mathrm{~ns})$ and have a higher probability of undergoing chemical reactions [3].

This quick overview is enough to notice that most photogenerated charge carriers do not undergo chemical reactions, but rather recombine, either radiatively or non-radiatively. Importantly, this implies that these processes chiefly dictate the lifetime of the charge carriers, and thus most kinetic analyses are centered in the recombination rate as a key variable to understand the photocatalytic activity of a given material.

\section{Charge Carrier Recombination Kinetics}

As noted, the understanding of photogenerated charge carriers and their kinetics is essential for the design and evaluation of a photocatalytically active material. A widely used method for the determination of the kinetics is transient absorption spectroscopy. Briefly, the photocatalyst material is excited with a laser pulse with a photon energy equal or larger than the band gap of the material. A spectrum is measured, with the help of an analyzing light, before and after the excitation, and from the difference of both spectra, the lifetime and the kinetics of the photogenerated charge carriers can be obtained. The reader is referred elsewhere for further details [10]. For measurements performed in diffuse reflectance mode, the difference is reported as the change of reflectance, $\Delta J$. In Fig. 2 the transient signal at $500 \mathrm{~nm}$ is shown for an anatase $\mathrm{TiO}_{2}$ photocatalyst powder (CristalACTiV $^{\text {TM }}$ PC105) after excitation with a $355 \mathrm{~nm}$ laser pulse. The initial $\Delta J$ value decreases rapidly until approximately $1 \mu \mathrm{s}$, while afterwards a significantly slower decrease can be observed. By taking the $\Delta J$ value at the same time point
Fig. 1 Excitation of a semiconductor particle with light with an energy equal or higher than the band gap and the following processes

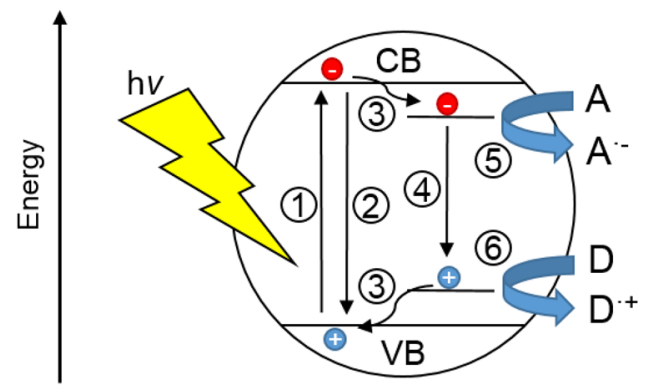

(1) Excitation

(2) Recombination of free charge carriers

(3) Trapping

(4) Recombination of trapped charge carriers

(5) Reduction

(6) Oxidation 

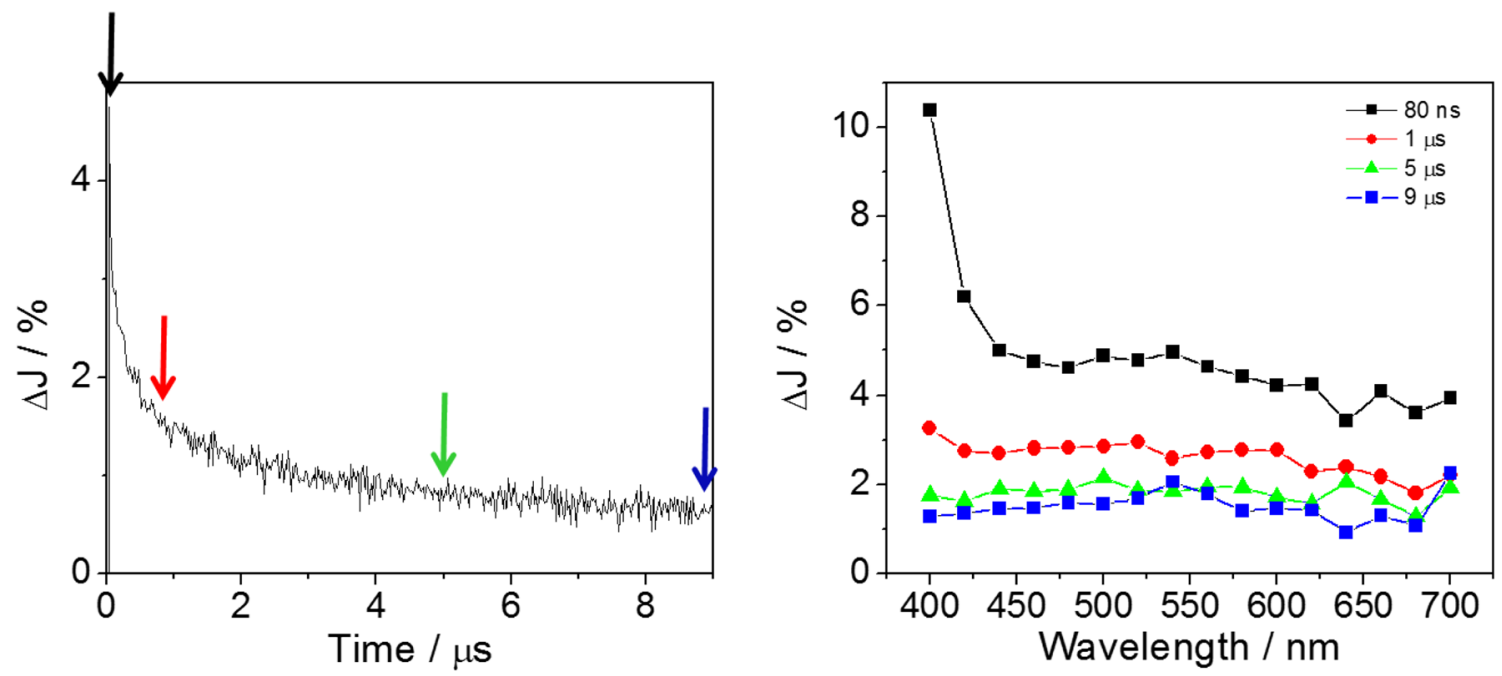

Fig. 2 Transient signal at $500 \mathrm{~nm}$ of $\mathrm{TiO}_{2}(\mathrm{PC} 105)$ after excitation with $355 \mathrm{~nm}$ (left) and transient absorption spectra at different times after the excitation (right). The colored arrows in the transient signal mark the time points at which the spectra were taken

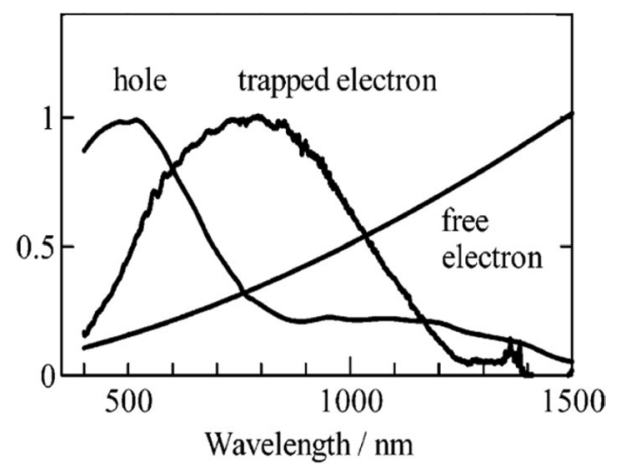

Fig. 3 Transient absorption spectra of holes, trapped electrons, and free electrons in $\mathrm{TiO}_{2}$. Reprinted with permission from reference [11]. Copyright 2014 American Chemical Society

for different detection wavelengths a transient absorption spectrum can be obtained. As displayed in Fig. 2 (right), as time increases, the overall intensity decreases.

Typically, a transient absorption spectrum can be split in different regions, with majority contributions from specific species. In the case of $\mathrm{TiO}_{2}$, this is illustrated in Fig. 3. The signal attributable to holes has a maximum at approximately $500 \mathrm{~nm}$, although it extends well into the near infrared. In the case of electrons, it is possible to differentiate between trapped and free ones, with the former showing a very broad feature peaking at ca. $700 \mathrm{~nm}$, and the latter showing a signal that increases continuously into the infrared. The entire spectra (Fig. 2, right) are, therefore, given by the combination of these signals. In turn, the decay in $\Delta J$ is related to the recombination of the photogenerated charge carriers, which leads to the disappearance of their signals.

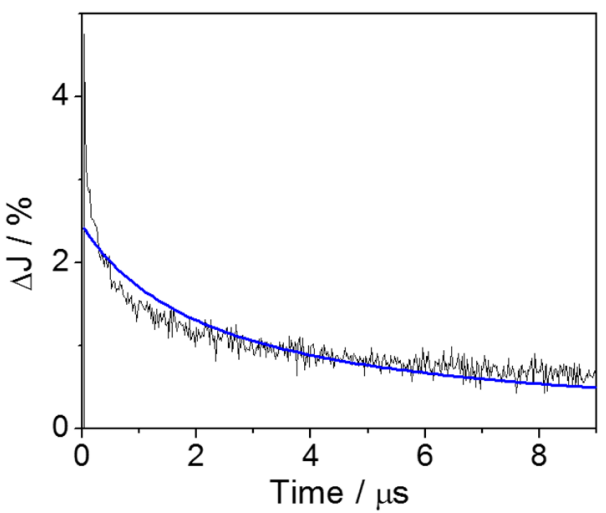

Fig. 4 Second order kinetics fit (blue) of the transient signal (black) at $500 \mathrm{~nm}$ of PC105 (anatase $\mathrm{TiO}_{2}$ ) after excitation with $355 \mathrm{~nm}$

The decay signals (Fig. 2, left) give information about the kinetics and the lifetime of the charge carriers. Several functions have been effectively employed to fit the decays, in order to obtain decay constants that can be related to recombination and/or reactive processes [10]. A frequently used function is that of the second-order kinetics model, which implies the bimolecular recombination of charge carriers. The function (Eq. 1) relates $\Delta J$, which is a function of time $t$, to the parameters $A$ (related to the initial height of the signal), B (baseline), and $\mathrm{k}$, the decay constant [7].

$\Delta J(t)=\frac{A}{1+A k t}+B$

In Fig. 4 the transient signal of PC105 at $500 \mathrm{~nm}$ (i.e. with similar contributions from electrons and holes) is shown together with a second order kinetics fit. The model 
Fig. 5 Reciprocal transient signal at $500 \mathrm{~nm}$ of PC105 (anatase $\mathrm{TiO}_{2}$, black) after excitation with $355 \mathrm{~nm}$ in the time scale of $1 \mathrm{~ms}$ (left) and $10 \mu$ s (right). The blue line and the red line represent different second order fits

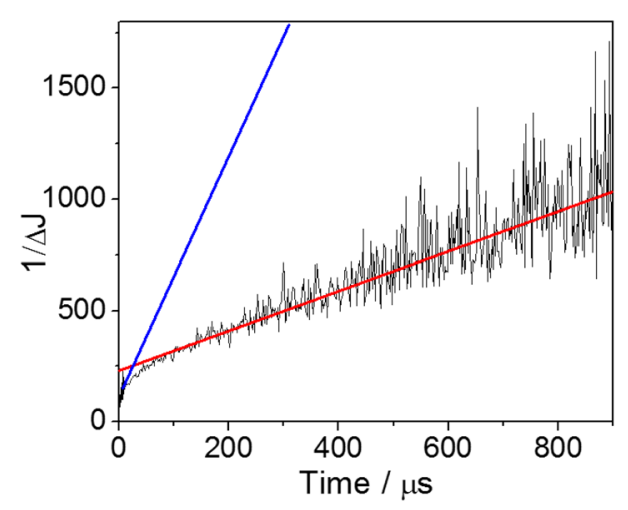

is not able to describe the obtained transient signal, and especially at early times there are significant differences between the fit function and the signal. A further problem of using this function is apparent after the linearization of the second order fit function (Fig. 5). If different time scales are considered, the application of the second order kinetics model yields different fit parameters. In other words, a fit function that describes the longer time ranges is not valid (at all) for the initial period after excitation. As illustrated in Fig. 5 (right panel), even a function that describes the 3 to $10 \mu$ s range strongly deviates for times shorter than $2 \mu \mathrm{s}$. The calculated kinetic parameters, thus, will strongly depend on the (arbitrarily chosen) time window; moreover, the second order kinetic model cannot be applied in broad time ranges.

A recently proposed model by Sieland et al. uses a fractals-based kinetic model for fitting the transient decays [7]. If a reaction is considered, where the diffusion is limited by the geometry, the rate constant $k$ is replaced by the fractal rate coefficient $k_{f}$, which is described by Eq. 2 , where $k_{2, f}$ is the fractal recombination constant, $t$ the time and $h$ the fractal dimension $[6,7]$. The fit model is obtained by replacing the rate constant in a second order model with Eq. 2, as shown in Eq. 3 [7]. The fractal dimension $h$ determines the time dependence of the rate constant [6]. In 3D homogenous media its value is 0 , which leads to classical kinetics and a time-independent rate constant, while diffusion-controlled reactions in fractal spaces lead to $h>0$ and time-dependent rate constants [6].

$k_{f}(t)=k_{2, f} t^{-h} \quad$ with $\quad 0 \leq h \leq 1$

$\Delta J(t)=\frac{A(1-h)}{(1-h)+A k_{2, f} t^{1-h}}$

Figure 6 shows the transient signal of PC105 at $500 \mathrm{~nm}$, as shown before, with the fractal kinetics fit. In contrast to the second order fit (Fig. 4) the fractal kinetics model is able to describe the whole time range of $10 \mu \mathrm{s}$. The fit model itself is also not only valid for short time scales, but also for times up to (at least) $100 \mu$ s (Fig. 7).

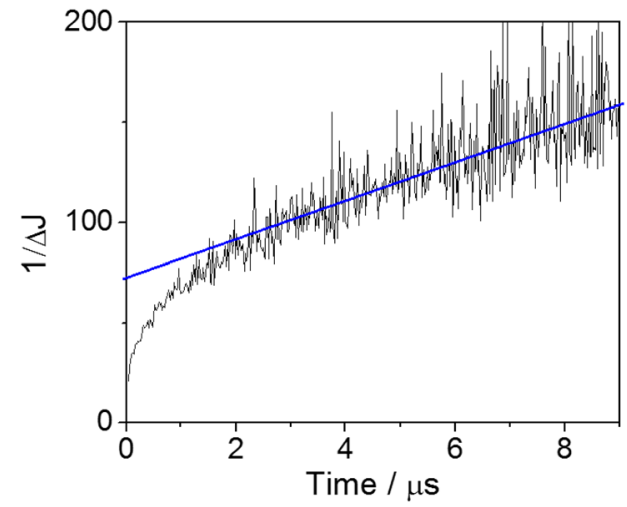

Fig. 6 Fractal kinetics fit (orange) of the transient reflectance signal at $500 \mathrm{~nm}$ of PC105 (anatase $\mathrm{TiO}_{2}$, black) after excitation with $355 \mathrm{~nm}$. Reproduced with permission from Ref. [12]

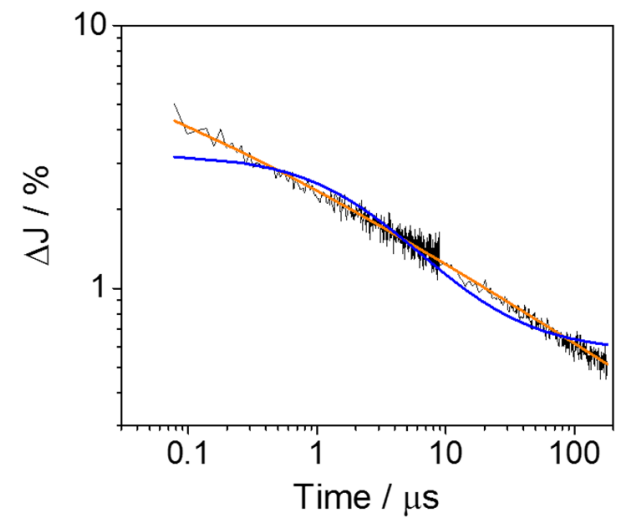

Fig. 7 Double logarithmic plot of the transient signal at $500 \mathrm{~nm}$ of PC105 (anatase $\mathrm{TiO}_{2}$, black) after excitation with $355 \mathrm{~nm}$ with the second order fit (blue) and the fractal kinetics fit (orange). Reproduced with permission from Ref. [12]

The application of the fractal model yields two parameters, the fractal recombination constant $k_{2, f}$ and the fractal parameter $h$. Table 1 contains the fit parameters for three different commercial $\mathrm{TiO}_{2}$ photocatalysts, including their BET surface area and primary particle size as determined 
Table 1 Obtained parameters from the fractal kinetics fit for commercial $\mathrm{TiO}_{2}$ powders [7]

\begin{tabular}{lllll}
\hline & $k_{2, f}$ (a.u.) & $h$ & $\begin{array}{l}\text { BET specific surface } \\
\text { area }\left(\mathrm{m}^{2} \mathrm{~g}^{-1}\right)\end{array}$ & $\begin{array}{l}\text { Primary } \\
\text { particle size } \\
(\mathrm{nm})\end{array}$ \\
\hline Kronos 1001 & 7.34 & 0.47 & 11 & 150 \\
CristalACTiVTM PC105 & 4.40 & 0.62 & 81 & 20 \\
CristalACTiVTM PC500 & 0.144 & 0.71 & 309 & 7.5 \\
\hline
\end{tabular}

by transmission electron microscopy (TEM). As expected, a lower primary particle size leads to a higher surface area [13]. The fractal recombination constant and the fractal parameter are related to the primary particle size as well: as it increases, the fractal recombination constant decreases, while the fractal parameter increases. The increasing fractal parameter can be further explained by the formation of agglomerates out of smaller particles for PC105 and PC500, which leads to more complex surfaces compared to the more even surface of Kronos 1001 given by its larger particle size [7].

Interestingly, the model is not only valid for $\mathrm{TiO}_{2}$, but it was shown to fit the transient signals of $\mathrm{WO}_{3}$ and $\mathrm{ZnFe}_{2} \mathrm{O}_{4}$ after excitation with a $355 \mathrm{~nm}$ laser pulse, as well (Fig. 8).

These examples have shown the suitability of the model for the fit of transient signals for powdered photocatalysts, but the model can be applied for fitting the signals of photocatalyst films, as well. A recent work showed that the lifetime and the decay constant of screen-printed $\mathrm{TiO}_{2}$ electrodes depend on the number of printed layers, and thus on the thickness of the films [14]. In Fig. 9 the same data is shown, but analyzed by means of the fractal kinetics model instead of the previously used second order model. The transient signals of the one-layer and four-layer electrodes, which are shown as an example, can be fitted very well with the fractal kinetics model, which is also the case for the electrodes with two and three layers. As described above, the fractal parameter is related to the roughness of a surface or particle. This can be proven as well for the $\mathrm{TiO}_{2}$ electrodes. The multilayer electrodes show a similar roughness, and also the values of the fractal parameter are comparable. In contrast, the surface roughness of the one-layer electrode is significantly lower; the fractal parameter displays the same behavior. The time-dependent fractal rate coefficients, calculated from the obtained fit parameters, are similar for the multilayer electrodes, while for the one-layer electrode the values are significantly higher. Thus, the transient signal decays faster for the one-layer electrode compared to the multilayer electrodes. These $\mathrm{TiO}_{2}$ electrodes are one example where both, the second order model and the fractal kinetics model, are suitable for fitting the transient decays, and they also lead to similar results [14]. However, by fitting with the fractal kinetics model the fractal parameter $h$ is obtained in addition, which is directly related to the surface roughness of the electrodes.

\section{Analysis of $\mathrm{TiO}_{2}$ Powder Mixtures}

By quantifying charge carrier recombination, transient absorption spectroscopy is a useful tool to explain the photocatalytic activity of a given set of materials. As a further evaluation of the fractal model, Sieland et al. [15] tested its suitability to explain the difference in the photocatalytic activity of $\mathrm{TiO}_{2}$ mixtures. In addition, another goal of this study was to evaluate the possibility of using $\mathrm{TiO}_{2}$ powders normally employed for paints; although they have a much smaller cost, they have not been considered for photocatalytic applications. Therefore, the commercial $\mathrm{TiO}_{2}$ photocatalysts PC500 and PC105, and the white pigment Kronos 1001, were chosen and compared. While the powders used as photocatalysts have similar primary particle sizes (in the
Fig. 8 Transient signals of $\mathrm{WO}_{3}$ at $440 \mathrm{~nm}$ (left) and of $\mathrm{ZnFe}_{2} \mathrm{O}_{4}$ (right) at $540 \mathrm{~nm}$ after excitation with $355 \mathrm{~nm}$ (black dots) and the fractal kinetics fit (orange line). Reproduced with permission from Ref. [12]
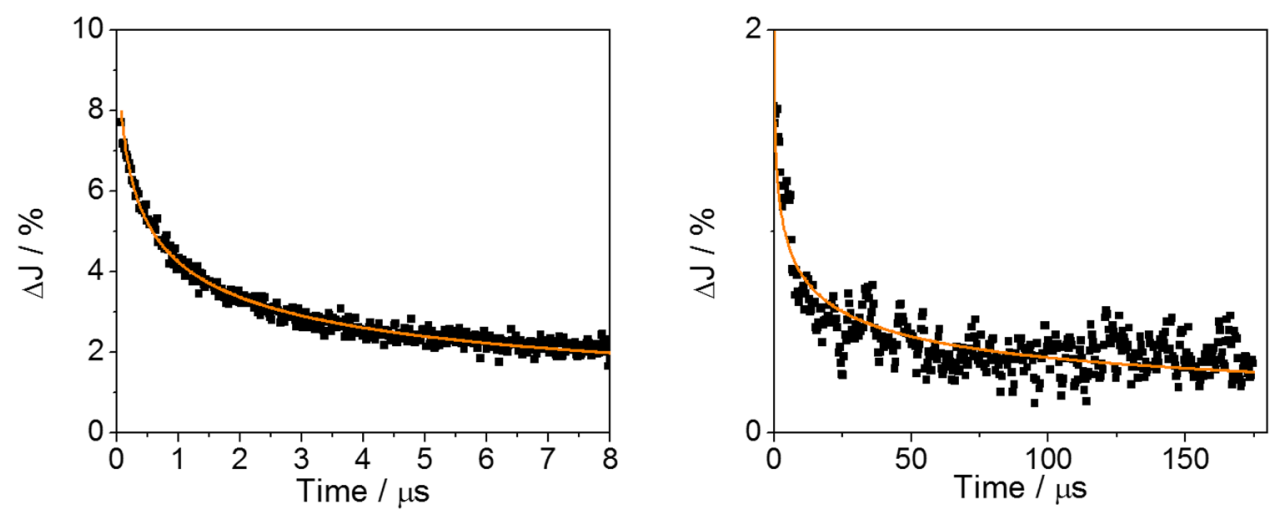

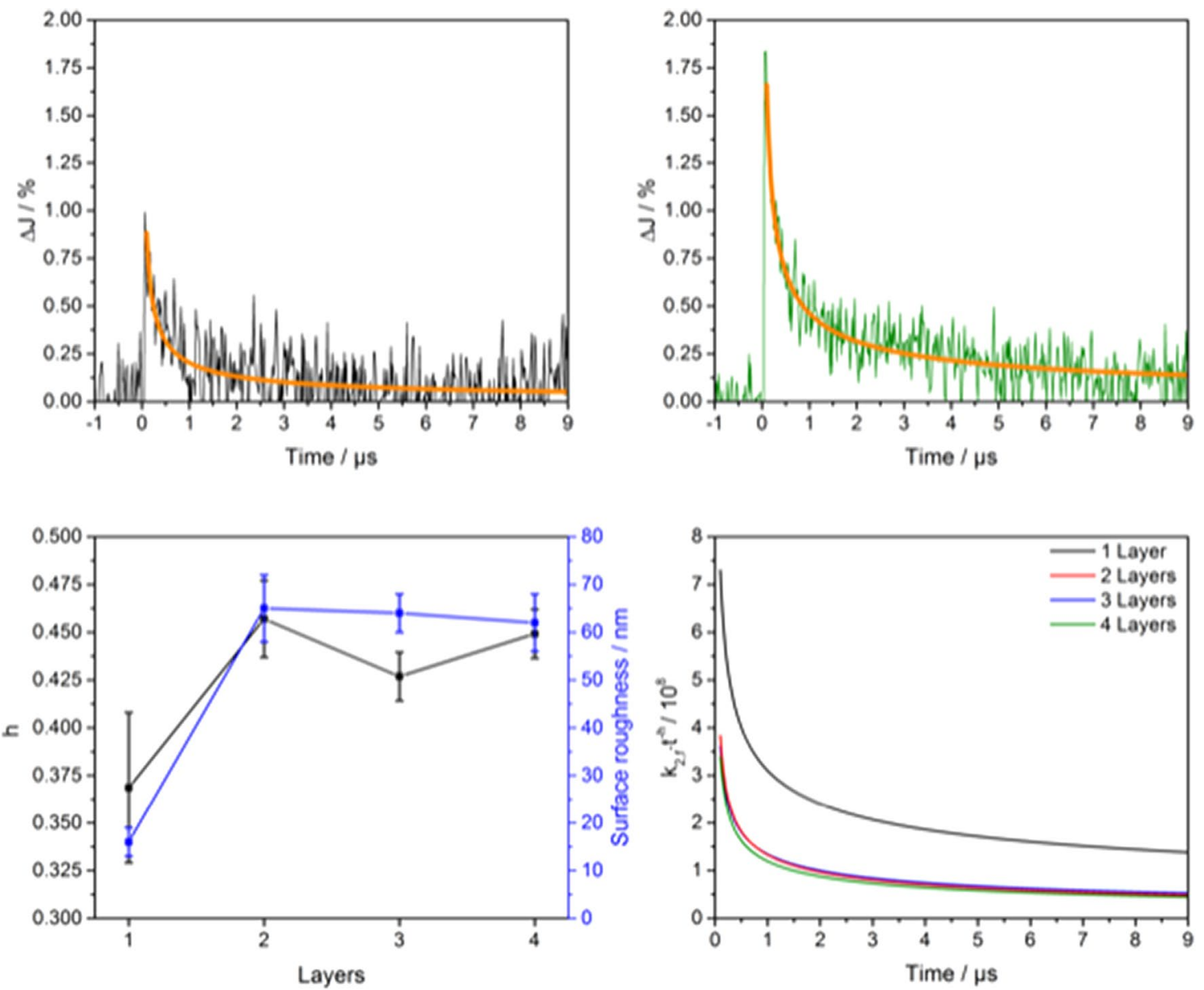

Fig. 9 Transient signals of screen-printed $\mathrm{TiO}_{2}$ electrodes with one layer (top left) and four layers (top right) at $600 \mathrm{~nm}$ after excitation with a $355 \mathrm{~nm}$ pulse. Obtained fractal parameters together with the

range $7-17 \mathrm{~nm}$ ), Kronos 1001 is composed of $125 \mathrm{~nm}$ primary particles (Fig. 10).

The procedure for creating the mixtures of the different $\mathrm{TiO}_{2}$ materials is shown in Fig. 11. Basically, a suspension of the smaller particles was created in ethanol and after sonication the larger particles were added. After a further sonication step, followed by centrifugation and drying, the mixed $\mathrm{TiO}_{2}$ powders were obtained.

The photocatalytic activity of the mixtures was investigated using standardized photocatalytic tests, that is, the degradation of acetaldehyde (ISO 22197-2) and the degradation of NO (ISO 22197-1) [15]. As shown in Fig. 12, the determined photonic efficiencies for the acetaldehyde degradation virtually show no dependency on the photocatalyst content for the mixtures of Kronos 1001 with either PC500 or PC105. This result is rather astonishing, since Kronos 1001 , usually employed as a white pigment, shows the same photocatalytic activity as the pure photocatalysts. This can be explained by two alternative explanations. First, it can

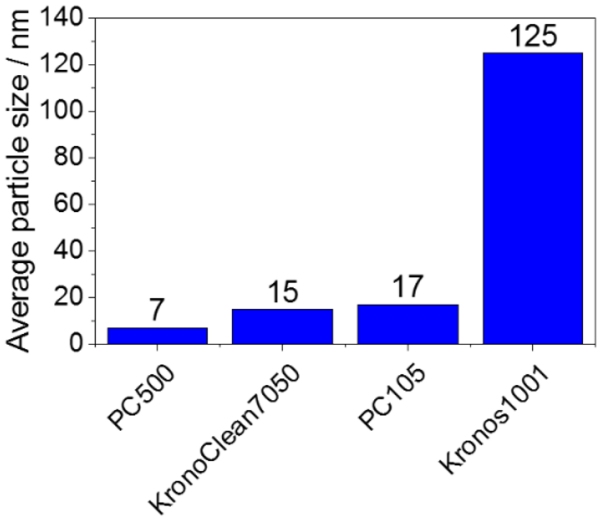

Fig. 10 Average particle sizes of the commercial photocatalysts PC500, KronoClean 7050, PC105, and of the white pigment Kronos 1001. Reproduced with permission from Ref. [15]—published by the PCCP Owner Societies

surface roughness (bottom left) and plot of the time-dependent rate constants (bottom right) for electrodes with one, two, three, and four screen-printed layers. Adapted with permission from Ref. [14] 
be considered that the smaller particles form agglomerates of similar sizes to those the bigger particles [15]. Therefore, for a degradation process that takes part exclusively on the outer surface of the agglomerates, a similar activity is expected [15]. Alternatively, it is possible that the different $\mathrm{TiO}_{2}$ materials induce different degradation pathways, fortuitously leading to similar activities [15].

In contrast to the acetaldehyde degradation, the photonic efficiency for the degradation of NO shows a different behavior in relation to the photocatalyst content (Fig. 13). While for the mixture of Kronos 1001 with PC500 a maximum was observed at a 30:70 composition, for the mixture of Kronos 1001 and PC105 a non-linear increase of the efficiency with the fraction of the latter was detected. The different photonic efficiencies are not explainable by the different surface areas of the mixtures (Fig. 14), which show a linear increase with an increasing photocatalyst content, which is expected due to the smaller particle size of the photocatalysts compared to the white pigment (Fig. 10).

The recombination rate constants of the $\mathrm{TiO}_{2}$ mixtures obtained with the fractal kinetics fit show in both cases an exponential decrease (Fig. 15) as the photocatalyst content increases. To perform a meaningful comparison, the fittings were performed by fixing the fractal coefficient to 0.5 [15], which leads to the unit $\mathrm{s}^{-0.5}$ for the fractal recombination constant. From these values, by taking the reciprocal, charge carrier lifetimes can be obtained (Fig. 16). The lifetimes show a trend that correlates well with the efficiencies of the NO degradation experiments (Fig. 16). For the mixtures of Kronos 1001 and PC500 a maximum for the lifetime is observed again at a 30:70 composition, while for the mixtures of Kronos 1001 and PC105 the
Fig. 11 Synthesis of the $\mathrm{TiO}_{2}$ mixtures via sonication. Reproduced with permission from Ref. [15]—published by the PCCP Owner Societies

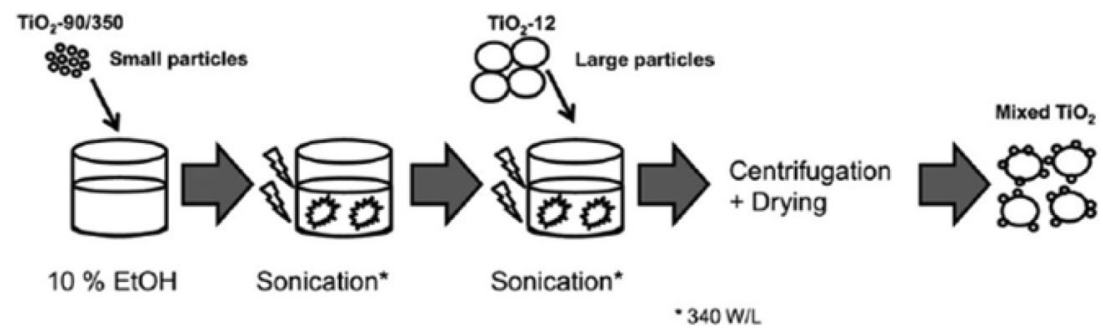

Fig. 12 Photonic efficiencies of the acetaldehyde degradation for PC500 (left) and PC105 (right) mixed with Kronos 1001. Reproduced with permission from Ref. [15]—published by the PCCP Owner Societies

Fig. 13 Photonic efficiencies of the NO degradation for PC500 (left) and PC105 (right) mixed with Kronos 1001. Reproduced with permission from Ref. [15] — published by the PCCP Owner Societies
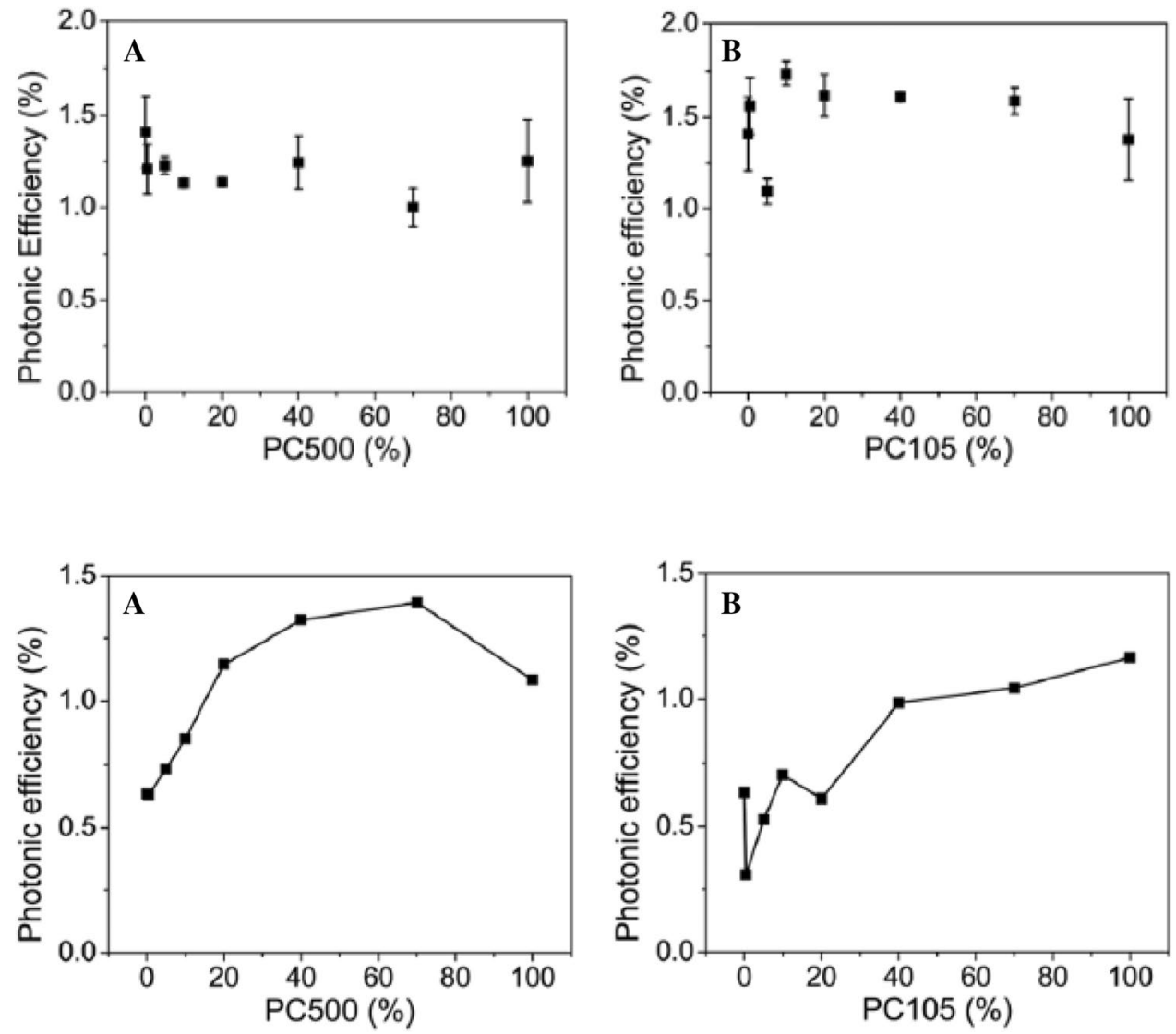
Fig. 14 BET surface area for PC500 (left) and PC105 (right) mixed with Kronos 1001. Reproduced with permission from Ref. [15]—published by the PCCP Owner Societies
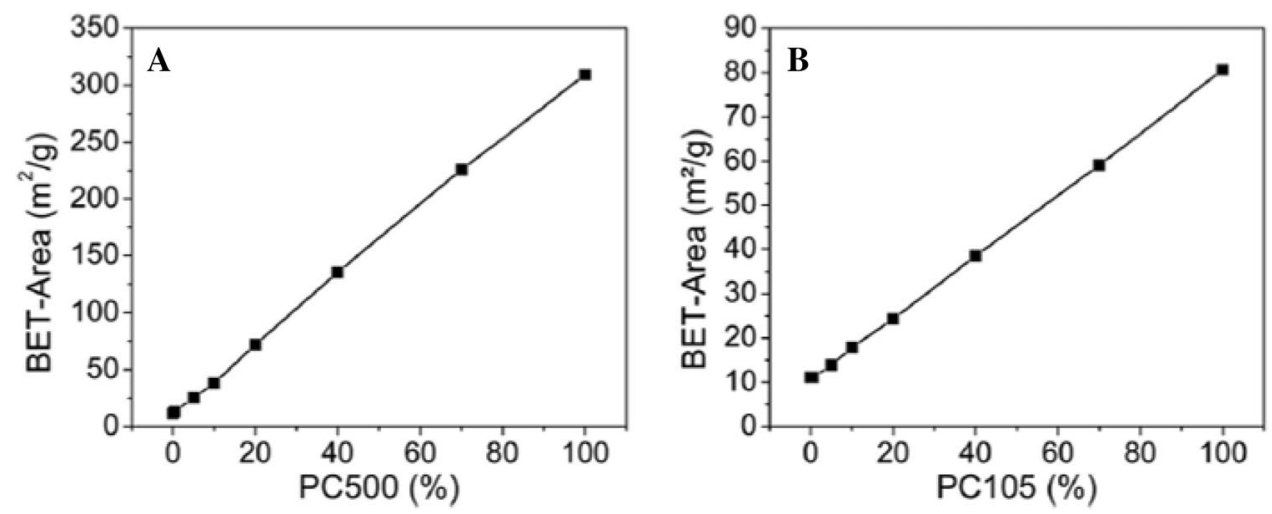

Fig. 15 Fractal recombination constants of the transient signal at $500 \mathrm{~nm}$ after excitation with $355 \mathrm{~nm}$ of the mixtures of Kronos 1001 with PC500 (left) and PC105 (right). Reproduced with permission from Ref. [15]published by the PCCP Owner Societies
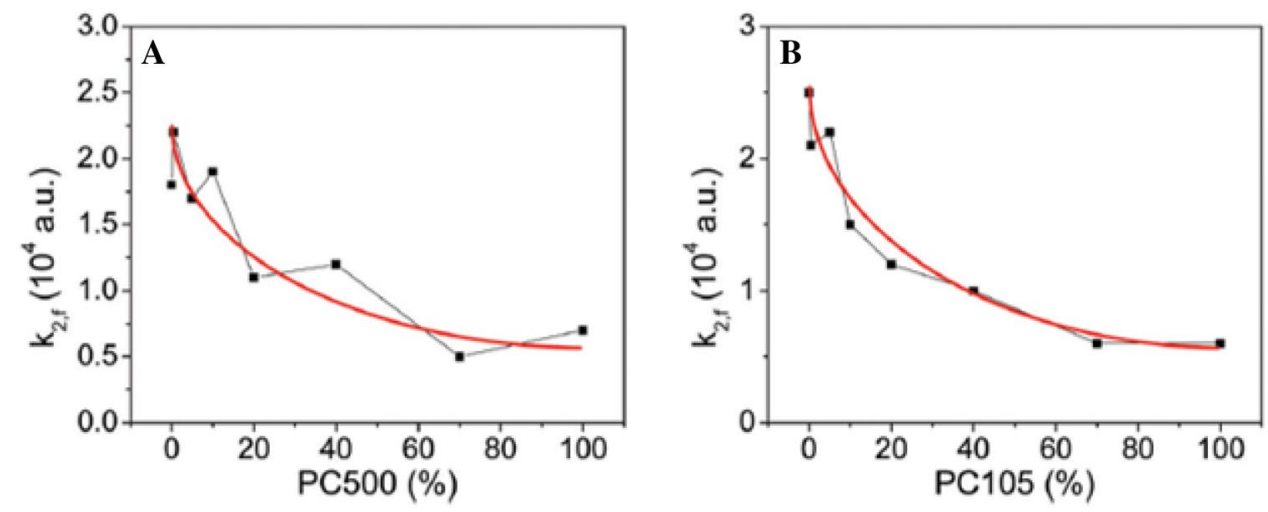

Fig. 16 Photonic efficiencies of the NO degradation (black) and the lifetime (reciprocal value of the fractal recombination constant, blue) of the mixtures of Kronos 1001 with PC500 (left) and PC105 (right). Reproduced with permission from Ref. [15] — published by the PCCP Owner Societies
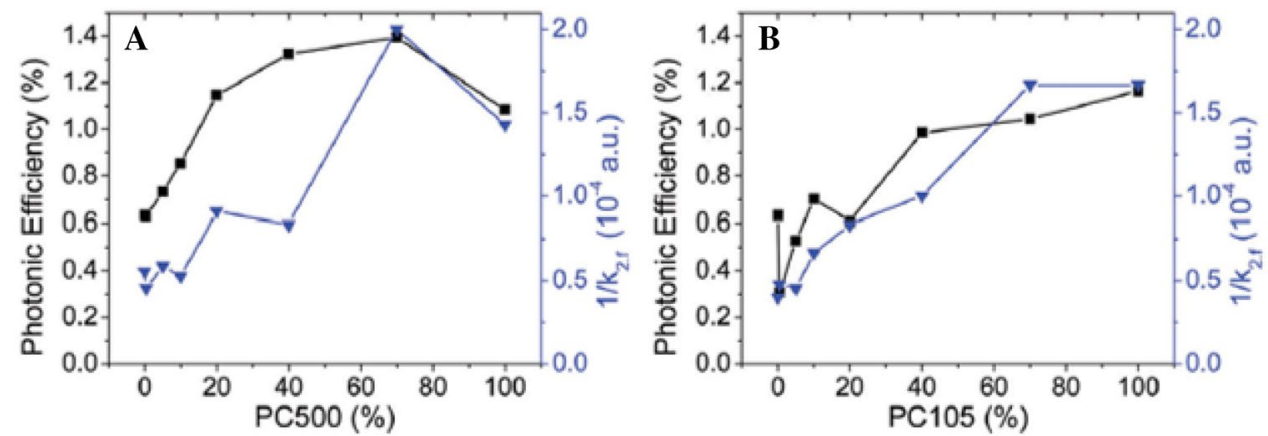

lifetime increases with the photocatalyst content in the same way as the efficiencies of the NO degradation. All in all, the lifetime of the photogenerated charge carriers has a higher impact on the photonic efficiencies of the NO degradation than the specific surface areas of the materials.

While the mixtures show a correlation between the charge carrier lifetime and the NO degradation, this is not the case for the acetaldehyde degradation. This can be explained by considering a much faster photocatalytic reaction of acetaldehyde in comparison to $\mathrm{NO}$, whereby the acetaldehyde degradation would be independent from the charge carrier lifetimes determined under inert gas atmospheres [15].

\section{Effect of Inert (?) Additives on Photocatalytic Activity}

The fractal model has also been effectively used to explain the photocatalytic activity of $\mathrm{TiO}_{2}$ powders mixed with (a priori inert) additives [16]. The mixtures were prepared by grinding the $\mathrm{TiO}_{2}$ powders together with the additive in solid form (Fig. 17). Mixtures of 0, 5, 10, 25, 50, and 100 vol\% photocatalyst with either $\mathrm{BaSO}_{4}, \mathrm{CaCO}_{3}$ or $\mathrm{Na}_{2} \mathrm{CO}_{3}$ were analyzed [16].

In Fig. 18 the photonic efficiency and the apparent quantum yield for the degradation of NO using mixtures 


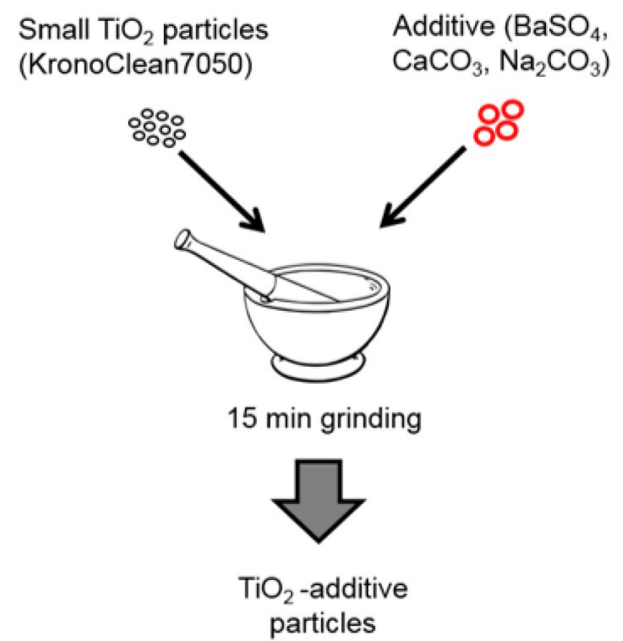

Fig. 17 Synthesis procedure for the mixtures of $\mathrm{TiO}_{2}$ with inert additives via grinding

of $\mathrm{TiO}_{2}$ with $\mathrm{BaSO}_{4}$ are shown. Interestingly, the activity increase does not follow a linear relation with the photocatalyst content, and is higher than expected, in the sense that large fractions of $\mathrm{BaSO}_{4}$ lead to a mild decrease in the photocatalytic activity. This trend can be explained by the differences in UV light absorption for all samples, where as well no linear relation between the photocatalyst content and the absorption can be seen (Fig. 18). The nearly constant apparent quantum yield for volume fractions of $\mathrm{TiO}_{2}$ between 0.2 and 1.0 could be explained by the scattering properties of $\mathrm{BaSO}_{4}$ (Fig. 19). Even while lower amounts of $\mathrm{TiO}_{2}$ are present, the light is scattered by the $\mathrm{BaSO}_{4}$ particles, allowing the excitation of $\mathrm{TiO}_{2}$ particles in deeper layers [16]. Therefore, even low volume fractions of $\mathrm{TiO}_{2}$ retain a high photocatalytic activity. On the other hand, too low fractions of $\mathrm{TiO}_{2}$ do lead to an activity decrease, since the content of $\mathrm{BaSO}_{4}$ is higher and a high fraction of UV light could be scattered out of the sample after multiple reflections [16].

Similarly, varying $\mathrm{TiO}_{2}$ contents showed no influence on the charge carrier recombination kinetics (Fig. 20). The

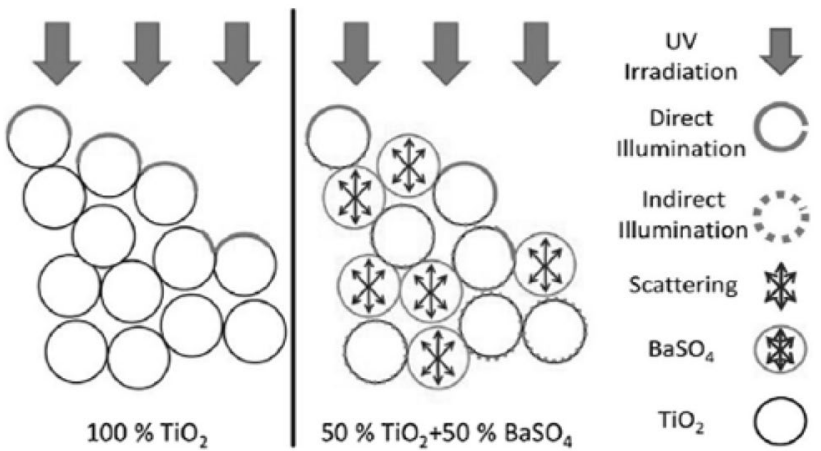

Fig. 19 Representations of the light absorption in bare $\mathrm{TiO}_{2}$ and in a mixture with $\mathrm{BaSO}_{4}$. While for the bare $\mathrm{TiO}_{2}$ just light in the particles in the front can be absorbed, for the sample with $\mathrm{BaSO}_{4}$ the light can be scattered deeper inside the material, enabling the absorption and excitation of more $\mathrm{TiO}_{2}$ particles. Reprinted from Ref. [16] with permission from Elsevier

fractal parameter was thereby fixed at the value of 0.5 for the fit of all decays of the $\mathrm{TiO}_{2}$ mixtures with the additives, since this value is the value that corresponds to pure $\mathrm{TiO}_{2}$ [16]. Moreover, the units of the rate constants determined with the fractal model depend on the value of $h$, and thus a meaningful comparison requires this parameter to be constant. We note however that preliminary fittings with variable $h$ parameters generally led to values close to 0.5 . The nearly constant recombination constant in dependence from the $\mathrm{TiO}_{2}$ content represents the expected behavior, since UV light is not able to excite $\mathrm{BaSO}_{4}$. Thus, just the reflection behavior of $\mathrm{BaSO}_{4}$ impacts the photocatalytic activity of the mixtures of $\mathrm{TiO}_{2}$ with $\mathrm{BaSO}_{4}$.

The apparent quantum yields for the degradation of $\mathrm{NO}$ over $\mathrm{TiO}_{2}$ mixtures with $\mathrm{CaCO}_{3}$ and $\mathrm{Na}_{2} \mathrm{CO}_{3}$, together with their UV absorption at $365 \mathrm{~nm}$, are shown in Fig. 21. The absorption of both sets of samples behaves similarly: it increases for higher fractions of $\mathrm{TiO}_{2}$, reaching a saturation value for $\mathrm{TiO}_{2}$ fractions larger than 0.5. Interestingly, for the mixtures of $\mathrm{TiO}_{2}$ with $\mathrm{CaCO}_{3}$ all different fractions show a similar activity, while for mixtures with $\mathrm{Na}_{2} \mathrm{CO}_{3}$ the activity rises markedly as the $\mathrm{TiO}_{2}$ content increases.
Fig. 18 Photonic efficiency for the degradation of $\mathrm{NO}$ together with the absorption at $365 \mathrm{~nm}$ (left) and apparent quantum yield (right) of the mixtures of $\mathrm{TiO}_{2}$ (KronoClean 7050) with $\mathrm{BaSO}_{4}$. Adapted from Ref. [16] with permission from Elsevier
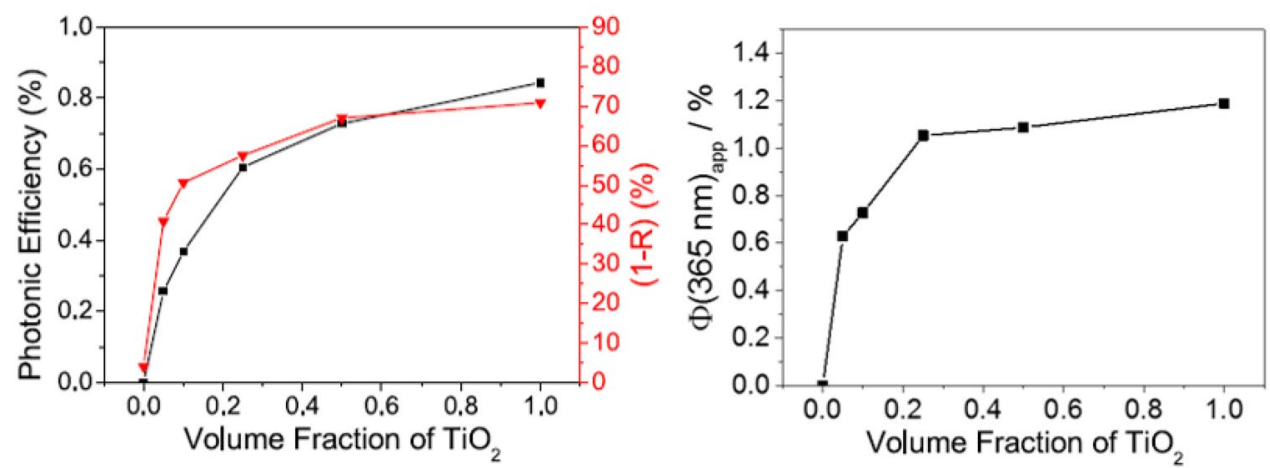


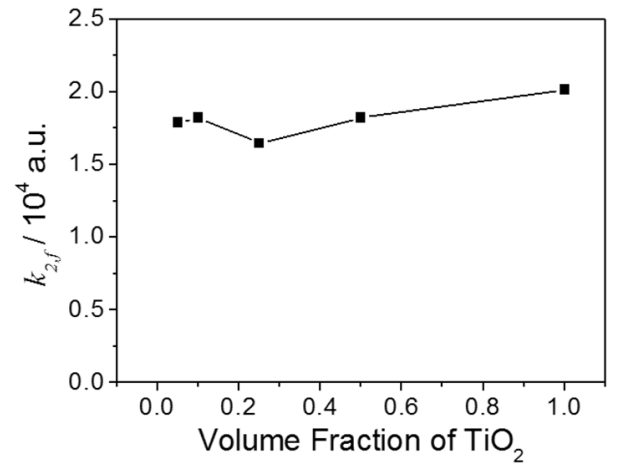

Fig. 20 Recombination constants obtained from the fractal kinetics fit of the transient signals at $500 \mathrm{~nm}$ after excitation with $355 \mathrm{~nm}$ of the mixtures of $\mathrm{BaSO}_{4}$ and $\mathrm{TiO}_{2}$. Adapted from Ref. [16] with permission from Elsevier

While for the mixtures of $\mathrm{TiO}_{2}$ with $\mathrm{CaCO}_{3}$ the charge carrier kinetics are uninfluenced by the $\mathrm{CaCO}_{3}$ content, charge carrier recombination in the mixtures with $\mathrm{Na}_{2} \mathrm{CO}_{3}$ is significantly faster (Fig. 22). These results match very well those of the $\mathrm{NO}$ degradation test: $\mathrm{TiO}_{2}$ mixtures with $\mathrm{CaCO}_{3}$ show similar recombination constants and photonic efficiencies for all mixtures, while for $\mathrm{Na}_{2} \mathrm{CO}_{3}$ even small additions increase the recombination rate, explaining the lowered photonic efficiencies [16].

The deleterious effect of sodium in $\mathrm{TiO}_{2}$ photocatalysts is well-known; it is particularly harmful in the case of selfcleaning surfaces, where thin films are employed and thus the substrate has a large influence $[17,18]$. In such cases, the use of sodium-containing glass substrates has been shown to considerably decrease the activity with respect to fused quartz ones. Two main arguments have been mentioned to explain the effect caused by the diffusion of sodium into the $\mathrm{TiO}_{2}$ phase: on the one hand, it inhibited the formation of anatase upon calcination; on the other, it introduced surface and bulk recombination centers [18]. Our transient absorption spectroscopy results offer, for the present system, support to the second hypothesis.

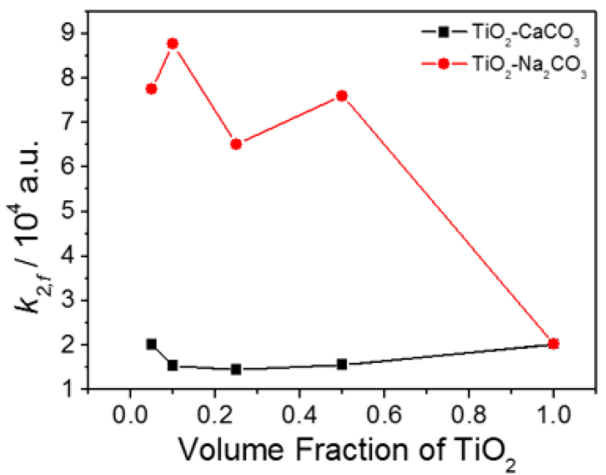

Fig. 22 Recombination constants obtained from the fractal kinetics fit of the transient signals at $500 \mathrm{~nm}$ after excitation with $355 \mathrm{~nm}$ of the mixtures of $\mathrm{TiO}_{2}$ with $\mathrm{CaCO}_{3}$ and $\mathrm{Na}_{2} \mathrm{CO}_{3}$. Adapted from Ref. [16] with permission from Elsevier

\section{Concluding Remarks}

In this short review we summarize recent results from our research group dealing with commercial $\mathrm{TiO}_{2}$ photocatalysts and their analysis with transient absorption spectroscopy. This technique gives valuable information on the primary processes occurring after excitation of the photocatalysts, the main one being charge carrier recombination. Although the analysis of such kinetics is not straightforward, we have proposed a new model based on the fractal characteristics of the materials that can be effectively employed in broad time windows. Importantly, the decay rate constants gave information on the recombination kinetics that is strongly correlated to the photocatalytic activity of the materials for the standardized NO degradation test. This insight has proved very valuable to analyze commercial photocatalyst powders in the presence of a priori inert additives: $\mathrm{BaSO}_{4}, \mathrm{CaCO}_{3}$, $\mathrm{NaCO}_{3}$, and $\mathrm{TiO}_{2}$-based white pigment. We found different effects depending on the chemical nature of the additive: while $\mathrm{BaSO}_{4}$ and $\mathrm{CaCO}_{3}$ are not detrimental to the photocatalytic activity, the addition of $\mathrm{NaCO}_{3}$ leads to a strong inhibition, explained by a much-increased recombination
Fig. 21 Absorption at $365 \mathrm{~nm}$ (left) and apparent quantum yields of the degradation of $\mathrm{NO}$ (right) for mixtures of $\mathrm{TiO}_{2}$ with $\mathrm{CaCO}_{3}$ and $\mathrm{Na}_{2} \mathrm{CO}_{3}$. Adapted from Ref. [16] with permission from Elsevier
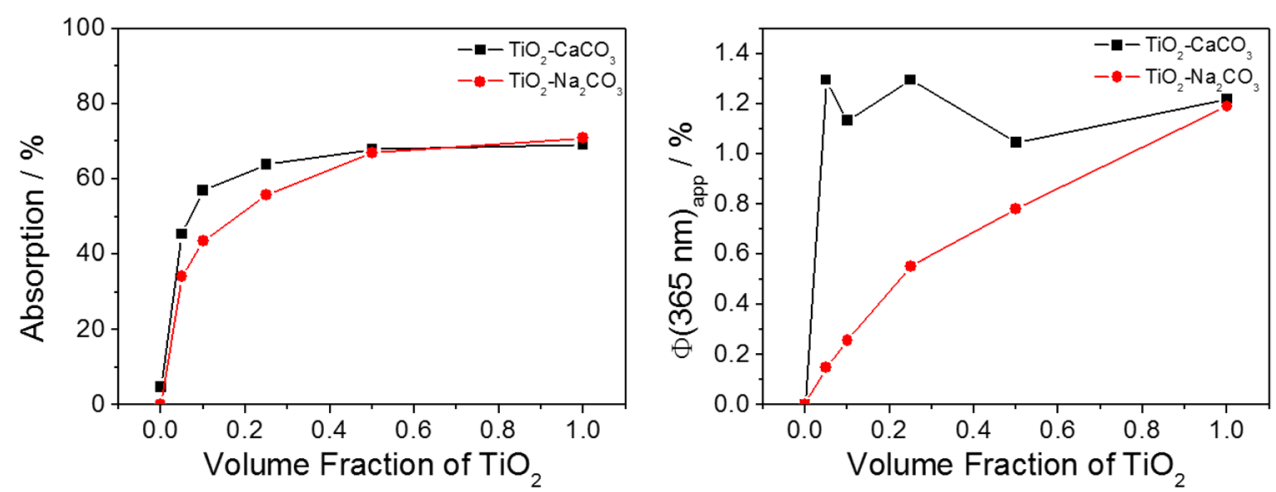
rate. Surprisingly, the incorporation of $\mathrm{TiO}_{2}$ commercially sold as a pigment into the mixtures did not decrease their photocatalytic activity for the degradation of acetaldehyde, despite a significant decrease in the specific surface areas.

Our results highlight the importance of evaluating potential additives in photocatalytic systems, and illustrates that low-cost powders sold as pigments can indeed be rather efficient photocatalysts. Both aspects are important towards industrial applications of $\mathrm{TiO}_{2}$-based photocatalytic systems.

Acknowledgements Open Access funding provided by Projekt DEAL. C.G. acknowledges financial support from the Leibniz Universität Hannover within the program "Wege in die Forschung II". M.C. is grateful to the Deutscher Akademischer Austauschdienst (DAAD) together with the Ministerio de Educación (Argentina) for his ALEARG scholarship. This work was supported by Saint-Petersburg State University via a research Grant ID 32706707.

\section{Compliance with Ethical Standards}

Conflict of interest The authors declare that they have no conflict of interest.

Open Access This article is licensed under a Creative Commons Attribution 4.0 International License, which permits use, sharing, adaptation, distribution and reproduction in any medium or format, as long as you give appropriate credit to the original author(s) and the source, provide a link to the Creative Commons licence, and indicate if changes were made. The images or other third party material in this article are included in the article's Creative Commons licence, unless indicated otherwise in a credit line to the material. If material is not included in the article's Creative Commons licence and your intended use is not permitted by statutory regulation or exceeds the permitted use, you will need to obtain permission directly from the copyright holder. To view a copy of this licence, visit http://creativecommons.org/licenses/by/4.0/.

\section{References}

1. Kamat PV, Christians JA (2015) Solar cells versus solar fuels: two different outcomes. J Phys Chem Lett 6:1917-1918. https://doi. org/10.1021/acs.jpclett.5b00897

2. Loeb SK, Alvarez PJJ, Brame JA, Cates EL, Choi W, Crittenden J, Dionysiou DD, Li Q, Li-Puma G, Quan X et al (2019) The technology horizon for photocatalytic water treatment: sunrise or sunset? Environ Sci Technol 53:2937-2947. https://doi.org/10.1021/ acs.est.8b05041

3. Schneider J, Matsuoka M, Takeuchi M, Zhang J, Horiuchi Y, Anpo M, Bahnemann DW (2014) Understanding $\mathrm{TiO}_{2}$ photocatalysis: mechanisms and materials. Chem Rev 114:9919-9986. https://doi.org/10.1021/cr5001892

4. Pinaud BA, Benck JD, Seitz LC, Forman AJ, Chen Z, Deutsch TG, James BD, Baum KN, Baum GN, Ardo S et al (1983) Technical and economic feasibility of centralized facilities for solar hydrogen production via photocatalysis and photoelectrochemistry. Energy Environ Sci 2013:6. https://doi.org/10.1039/c3ee40831k
5. Hakki A, Schneider J, Bahnemann D (2016) Understanding the chemistry of photocatalytic processes. In: Schneider J, Bahnemann D, Ye J, Li Puma G, Dionysiou DD (eds) Photocatalysis: fundamentals and perspectives. The Royal Society of Chemistry, London, pp 29-50

6. Kopelman R (1988) Fractal reaction kinetics. Science 241:16201626. https://doi.org/10.1126/science.241.4873.1620

7. Sieland F, Schneider J, Bahnemann DW (2017) Fractal charge carrier kinetics in $\mathrm{TiO}_{2}$. J Phys Chem C 121:24282-24291. https ://doi.org/10.1021/acs.jpcc.7b07087

8. Plonka A (1988) Chapter 3. Dispersive kinetics in condensed phases. Annu. Rep. Prog. Chem. Sect. C 85:47-75. https://doi. org/10.1039/pc9888500047

9. Grela MA, Colussi AJ (1996) Kinetics of stochastic charge transfer and recombination events in semiconductor colloids. Relevance to photocatalysis efficiency. J. Phys. Chem. 100:18214-18221. https ://doi.org/10.1021/jp961936q

10. Günnemann C, Curti M, Schneider J, Bahnemann DW (2020) Dynamics of photoinduced bulk and surface reactions involving semiconductors characterized by time resolved spectroscopy techniques (2015-2018). In: Albini A, Protti S (eds) Photochemistry, vol 47. The Royal Society of Chemistry, London, pp 122-158

11. Yoshihara T, Katoh R, Furube A, Tamaki Y, Murai M, Hara K, Murata S, Arakawa H, Tachiya M (2004) Identification of reactive species in photoexcited nanocrystalline $\mathrm{TiO}_{2}$ films by wide-wavelength-range (400-2500 Nm) transient absorption spectroscopy. J Phys Chem B 108:3817-3823

12. Sieland F (2018) Fractal charge carrier recombination kinetics in photocatalytic systems. Gottfried Wilhelm Leibniz Universität, Hannover

13. Feldhoff A, Mendive C, Bredow T, Bahnemann D (2007) Direct measurement of size, three-dimensional shape, and specific surface area of anatase nanocrystals. ChemPhysChem 8:805-809. https://doi.org/10.1002/cphc.200700084

14. Günnemann C, Curti M, Eckert JG, Schneider J, Bahnemann DW (2019) Tailoring the photoelectrochemical activity of $\mathrm{TiO}_{2}$ electrodes by multilayer screen-printing. ChemCatChem 11:64396450. https://doi.org/10.1002/cctc.201901872

15. Sieland F, Schneider J, Bahnemann DW (2018) Photocatalytic activity and charge carrier dynamics of $\mathrm{TiO}_{2}$ powders with a binary particle size distribution. Phys Chem Chem Phys 20:81198132. https://doi.org/10.1039/C8CP00398J

16. Sieland F, Duong NA-T, Schneider J, Bahnemann DW (2018) Influence of inorganic additives on the photocatalytic removal of nitric oxide and on the charge carrier dynamics of $\mathrm{TiO}_{2}$ powders. J Photochem Photobiol A 366:142-151. https://doi.org/10.1016/j. jphotochem.2018.01.036

17. Paz Y, Luo Z, Rabenberg L, Heller A (1995) Photooxidative selfcleaning transparent titanium dioxide films on glass. J Mater Res 10:2842-2848. https://doi.org/10.1557/JMR.1995.2842

18. Paz Y, Heller A (1997) Photo-oxidatively self-cleaning transparent titanium dioxide films on soda lime glass: the deleterious effect of sodium contamination and its prevention. J Mater Res 12:27592766. https://doi.org/10.1557/JMR.1997.0367

Publisher's Note Springer Nature remains neutral with regard to jurisdictional claims in published maps and institutional affiliations. 